\title{
Periodic orbit theory of two coupled Tchebyscheff maps
}

\author{
C. P. Dettmann ${ }^{1 *}$ and D. Lippolis ${ }^{1,2 \dagger}$ \\ 1. Department of Mathematics, University of Bristol, \\ University Walk, Bristol, BS8 1TW, United Kingdom \\ 2. Dipartimento di Fisica, Universitá di Bologna, \\ viale C. Berti Pichat 6/2, Bologna 40127, Italy
}

November 21, 2018

\begin{abstract}
Coupled map lattices have been widely used as models in several fields of physics, such as chaotic strings, turbulence, and phase transitions, as well as in other disciplines, such as biology (ecology, evolution) and information processing. This paper investigates properties of periodic orbits in two coupled Tchebyscheff maps. Then zeta function cycle expansions are used to compute dynamical averages appearing in Beck's theory of chaotic strings. The results show close agreement with direct simulation for most values of the coupling parameter, and yield information about the system complementary to that of direct simulation.
\end{abstract}

\section{Introduction}

Coupled map lattices (CML) are dynamical systems with discrete space and time and continuous state variables. Their most common form in applications is the diffusively coupled model

$$
\Phi_{n+1}^{(i)}=(1-a) f\left(\Phi_{n}^{(i)}\right)+(a / 2)\left[g\left(\Phi_{n}^{(i+1)}\right)+g\left(\Phi_{n}^{(i-1)}\right)\right]
$$

where $n$ is a discrete time step, $i$ is a lattice point with a periodic boundary condition, $f(\Phi)$ the local map, $g(\Phi)$ the coupling and $a$ a continuous parameter. They prove particularly useful in the investigation of chaotic dynamics of spatially extended systems (spatiotemporal chaos), but have also been employed in chemical, biological and engineering modelling, as well as pattern formation. The discrete structure in space and time can either be the approximation of

*Carl.Dettmann@bris.ac.uk

${ }^{\dagger}$ Future address: Department of Physics, Georgia Institute of Technology, Atlanta GA 30332, USA 
a less tractable continuous system (as in turbulence), or may arise naturally in applications (as in population dynamics of discrete colonies of organisms). Refs. 1, 2, 3, 4, give a good overview of early work to 1998 including the above applications; recent developments include advances in the mathematics of the relevant Banach spaces and transfer operators [5, 6] as well as ever widening applications; examples include ecology [7, 8, 9, neurons [10, 11, traffic flow [12] cryptography [13, 14] and field theory [15, 16].

From the point of view of understanding the dynamics of coupled chaotic maps, the most common CML studied are where the local dynamics $f$ is quadratic (the "logistic" map); this class of one dimensional maps is now reasonably well understood. The simplest example of a quadratic map is the second degree Tchebyscheff polynomial $f(\Phi)=T_{2}(\Phi)=2 \Phi^{2}-1$ which is well known to be conjugated to a piecewise linear map with complete binary symbolic dynamics, and hence exactly solvable.

In this paper we consider diffusively coupled Tchebyscheff maps (see Sec. 2.2 for details) of degree 2 and 3. These have been previously used by Beck [15] 16] in some interesting but unconfirmed ideas concerning dynamical generation of noise for stochastic quantisation leading to predictions of parameters in the standard model of particle physics. One of the authors [17] subsequently showed that four of Beck's six CML models exhibit stable synchronised time periodic states at arbitrarily small values of the coupling parameter $a$, albeit with small basins of attraction. The motivation for considering these models derives not only from possible applications, but also theoretical simplicity in that the uncoupled state is fully chaotic and exactly solvable, the small coupling limit has interesting structure, and the equations (being low degree polynomials) allow many exact calculations.

Here we apply classical periodic orbit theory [18 19 to a lattice of two coupled Tchebyscheff maps. This theory allows very precise computations of long time properties (averages, Lyapunov exponents, dimensions, decay of correlations, etc.) of chaotic systems, but has been mostly restricted to low dimensional systems in the past. This paper contains the first steps towards developing a high dimensional periodic orbit theory, making use of the properties of Tchebyscheff maps to locate a large number of periodic orbits. If the Beck theory turns out to be true, the insight gained regarding these CML will be invaluable in developing periodic orbit based computation methods which are potentially far more precise than direct simulation, and which are required in particle physics. More generally, a periodic orbit theory of CML will allow a far greater precision and insight into the many spatiotemporal systems discussed at the beginning than is currently available using direct methods.

In order to achieve our immediate goal of computing Beck's observables on a two site lattice, we first need to look for periodic orbits of (4) by using appropriate numerical methods and study some of their properties, as described in the following sections. The locations, the stability and the number of periodic orbits of such maps depend on the value of $a$ and current results do not allow prediction of these changes in detail. As we discuss in section 2, unexpected phenomena take place as soon as the coupling parameter $a$ is increased from 
0 , for example a few periodic points suddenly move off the region $\Phi^{i} \in[-1,1]$, which we consider for our analysis. As $a$ is increased from, periodic points drift on the domain $\Phi^{i} \in[-1,1]$, moving into the complex plane and then reappearing on the real axis as $a$ is increased further. The stability of periodic orbits is also heavily affected by the value of the coupling and stable and/or almost stable orbits appear for certain $a$ 's. Such behaviour has important consequences for the convergence of cycle expansions 19, as discussed in section 3. In fact both the rate and the kind of convergence of any average we compute on the lattice strongly depends on the value of the coupling parameter. Still in the same section we address the applications of equations (4) to the theory of Beck. As pointed out by one of the authors [17, the system (4) does not show ergodicity for all values of $a$, therefore direct simulation is not always guaranteed to be reliable in computing averages over the lattice. That is one reason why we investigate the efficiency of averaging over the periodic points. When it is possible to achieve a significant precision for the zeta function (see sec. 3.1), we have computed the average of some string potentials defined in sec. 3.2, and then compared the results with direct simulation on the same lattice. Conclusions and outlook are presented in section 4 .

\section{Periodic solutions}

\subsection{Finding periodic orbits}

The first problem is to find periodic solutions to the map (4) up to the highest possible period. A number of numerical methods can be employed for that purpose. In our case the quickest one seems to be the inverse iteration method, as follows: Let $x^{\prime}=f(x)$ be a map whose cycles are all expanding ${ }^{1}$, then it follows that its inverse $x^{\prime}=f^{-1}(x)$ has only stable periodic orbits, which can easily be found by iteration. A different symbol sequence (where symbols are associated with branches of the multivalued inverse) is to be used for every orbit [19. Unfortunately this method cannot be applied a priori, unless we are certain that the map has only expanding cycles. It turns out that the system (4) has got a few periodic orbits whose Jacobian has one stable and one unstable eigenvalue (see sec 2.3). This makes it impossible for inverse iteration to find them, since we cannot guess initial conditions that lie right on the unstable manifold.

A different approach to the problem is that to find the zeros of the equation $f^{n}(x)-x=0$, where $n$ is the period in question. The multipoint Newton's method [19] is believed to be one of the best algorithms for that purpose. It has the advantage of converging relatively fast to the solution, provided that sufficiently accurate initial conditions are given. A cycle of period $n$ is a root of

\footnotetext{
${ }^{1}$ they have no marginal or contracting directions
} 

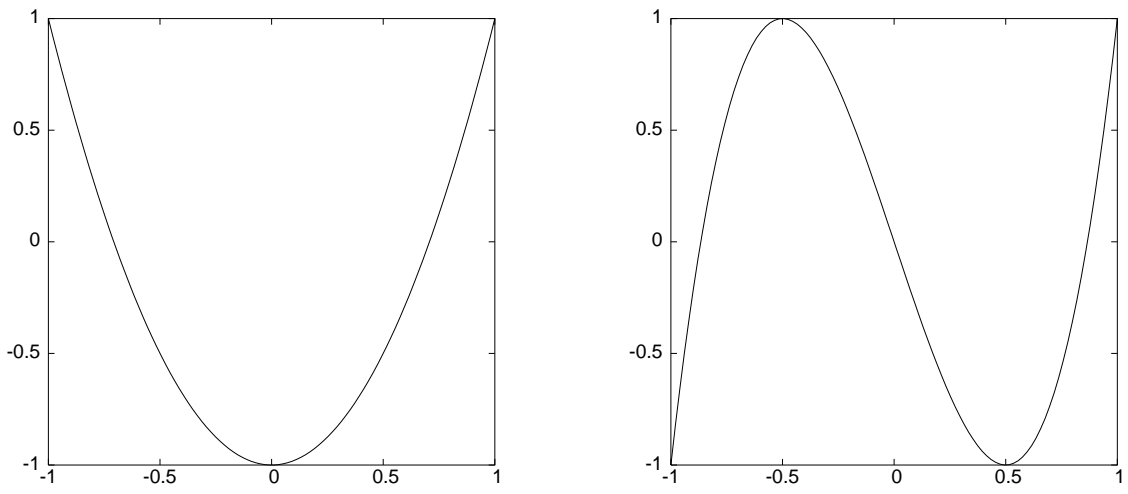

Figure 1: $T_{2}$ and $T_{3}$ polynomials as defined in (3).

the vector function $F$ :

$$
F(x)=F\left(\begin{array}{c}
x_{1} \\
x_{2} \\
\cdots \\
x_{n}
\end{array}\right)=\left(\begin{array}{c}
x_{1}-f\left(x_{n}\right) \\
x_{2}-f\left(x_{1}\right) \\
\ldots \\
x_{n}-f\left(x_{n-1}\right)
\end{array}\right)
$$

The iteration we need in order to approach the roots has the form

$$
\frac{d}{d x} F(x)\left(x^{\prime}-x\right)=-F(x) \Rightarrow x^{\prime}=x-\left[\frac{d}{d x} F(x)\right]^{-1} F(x)
$$

Therefore, after a certain number of iterations we expect to find one root. Of course the method has to be performed every time with different initial conditions, until we are sure all the solutions have been found. The issue concerning the choice of initial conditions is a delicate one, because in principle it is difficult to guess the initial points on a 2-dimensional plane accurately. Nevertheless, it is useful to remember that we are dealing with weakly coupled maps $(a \ll 1)$. For that reason we expect the periodic points to be relatively close to those of the uncoupled system $(a=0)$, that can be found analytically (see sec. 2.2) and hence exactly.

\subsection{Uncoupled system}

The spatially uncoupled dynamics of Tchebyscheff maps is given by

$$
\Phi_{n+1}^{i}=T_{N}\left(\Phi_{n}^{i}\right)
$$


where $T_{N}$ is the $N$ th Tchebyscheff polynomial, defined in general as

$$
T_{N}(\Phi)=\cos (N \arccos \Phi)
$$

of which we consider the first two, namely

$$
\begin{gathered}
T_{2}(\Phi)=2 \Phi^{2}-1 \\
T_{3}(\Phi)=4 \Phi^{3}-3 \Phi
\end{gathered}
$$

The general (coupled) Tchebyscheff maps have the form

$$
\Phi_{n+1}^{i}=(1-a) T_{N}\left(\Phi_{n}^{i}\right)+s \frac{a}{2}\left(T_{N}^{b}\left(\Phi_{n}^{i-1}\right)+T_{N}^{b}\left(\Phi_{n}^{i+1}\right)\right)
$$

where $a$ is the string coupling parameter, $s$ can be \pm 1 and $b$ can take on the values 1 and 0 , determining respectively advanced coupling $\left(T_{N}^{1}(\Phi)=T_{N}(\Phi)\right.$, "A") or backward coupling $\left(T_{N}^{0}(\Phi)=\Phi\right.$, "B"). We have then six models $2 A^{+}$, $2 A^{-}, 2 B^{+}, 2 B^{-}, 3 A^{+}$and $3 B^{+}$. The other two $N=3$ maps are equivalent to these by a trivial symmetry.

The model (4), becomes

$$
\begin{aligned}
& x_{n+1}=(1-a) T_{N}\left(x_{n}\right)+s a T_{N}^{b}\left(y_{n}\right) \\
& y_{n+1}=(1-a) T_{N}\left(y_{n}\right)+\operatorname{saT}_{N}^{b}\left(x_{n}\right)
\end{aligned}
$$

on a two site lattice. In this section we consider the uncoupled case $a=0$, in which the system (56) reduces to

$$
x^{\prime}=T_{N}(x) \quad y^{\prime}=T_{N}(y)
$$

This is useful as there is a close correspondence between orbits at $a=0$ and orbits with $a>0$. This correspondence is used to find orbits in the coupled dynamics using the uncoupled orbits as starting points for the multipoint Newton algorithm. The correspondence is not exact - the $N^{2 n_{p}}$ degree polynomial equation giving the periodic condition always has this number of (possibly degenerate) solutions, however for $a>0$ some of these solutions are not in the range $[-1,1]$ or are complex, and hence not relevant to the dynamics. This is discussed further in the next section.

We begin first with some definitions. A periodic point of length $n_{p}$ is a solution of $f^{n_{p}}(x)=x$; as discussed above, there are at most $N^{2 n_{p}}$ of them. A periodic orbit is the orbit $\left\{f^{j}(x)\right\}, j=0,1, \ldots n_{p}-1$ of a periodic point. A prime orbit is a periodic orbit which is not the repeat of a shorter orbit, that is, $f^{j}(x)=x$ only if $j$ is a multiple of $n_{p} .(x, y)$ is a periodic point of length $n_{p}$ of the uncoupled system if and only if $x$ and $y$ are both periodic points of length $n_{p}$ of the map $T_{N}$. It is well known [16] that the $T_{N}$ maps are conjugate to piecewise linear maps with complete $N$-ary symbolic dynamics, by the transformation $x=\cos \pi \theta$. This leads to exact expressions for all the periodic points of length $n_{p}$, namely $x=\cos \left[2 j \pi /\left(N^{n_{p}} \pm 1\right)\right]$ with $0 \leq j \leq N^{n_{p}} / 2$. From these we enumerate all the prime orbits of the uncoupled two dimensional dynamics, 
eliminating cyclic permutations which correspond to points on the same orbit, and repeats of shorter orbits.

As an example we consider $N=n_{p}=2$. Let symbol 0 (1) denote $x \in$ $[-1,0]$ (respectively $x \in(0,1])$; in general there are $N$ symbols. In the symbolic representation of periodic points, the spatial variable ( $i$ in Eq. (1)) will increase to the right, and the temporal variable ( $n$ in Eq. (1)) will increase downwards. The periodic points of the one dimensional map are

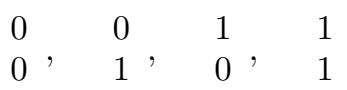

leading to 16 periodic points in the two dimensional map, classified as six prime orbits

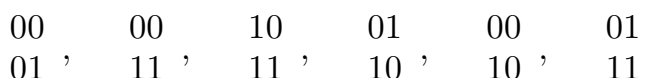

their cyclic permutations

\begin{tabular}{|c|c|c|}
\hline & 11 & 10 \\
\hline 0 & $10^{\prime}$ & 01 \\
\hline
\end{tabular}

and four repeated period one orbits

$\begin{array}{llll}00 & 01 \\ 00 & 01\end{array}, \begin{array}{ll}10 \\ 10\end{array}, \quad 11$

\subsection{Symmetry}

Until now we have not considered the symmetry of the system. This is particularly important for cycle expansions [19, 20, for which a correct treatment of symmetry significantly accelerates convergence of the expansions. An uncoupled lattice has symmetry group $S_{X} \times G^{X}$ where $X$ is the number of lattice sites (equal to two in this paper), $S_{X}$ is the symmetric group, of order $X$ !, corresponding to permutations of the lattice points, and $G$ is the symmetry group of the dynamics. A symmetrically coupled lattice with $X>2$ has symmetry group $D_{X}$, the dihedral group, of order $2 X$, corresponding to spatial cyclic permutations and reflections of the lattice sites. In the case $X=2$ the cyclic shift and reflection are equivalent, so the group is $C_{2}$, the cyclic group. Remnants of the internal symmetry group $G$ may or may not survive, depending on the nature of the coupling. For $X>3$ the coupling breaks the permutation symmetry, reducing the order of the symmetry group (ignoring internal symmetries), from $X$ ! to $2 X$ [21].

Each symmetry operation permutes periodic points. If the image of a periodic point lies in the same periodic orbit, that orbit is called symmetric, and is invariant under a cyclic subgroup of symmetry operations. If it does not, the new orbit is equivalent to the first under the symmetry, and shares many of the same properties, in particular stability and averages invariant under the symmetry. It is also possible for a periodic point to be a fixed point of the symmetry 
transformation, in which case its orbit is called a boundary orbit. In the presence of symmetry, the best strategy is desymmetrisation, in which all images of a phase space point are considered equivalent, and so may be described by a representative point on a subset of the original phase space called the fundamental domain. Equivalent orbits in the full dynamics correspond to a single orbit in the desymmetrised dynamics, and symmetric orbits in the full dynamics correspond to repeats of shorter orbits in the desymmetrised dynamics. Thus the enumeration of prime orbits up to a given period in the desymmetrised dynamics contains much more information than the enumeration up to the same period in the original dynamics, hence the accelerated convergence of cycle expansions.

We continue with the previous example, considering the $N=2$ maps, which have no internal symmetry. Thus the symmetry group is the cyclic group $C_{2}$, with the nontrivial element corresponding to $(x, y) \rightarrow(y, x)$. The fundamental domain can be taken as $x \geq y$, from which the full domain is obtained by taking symmetry operations. The boundary satisfies $x=y$. Now the prime orbits of period two in the desymmetrised dynamics are classified as follows. There are two asymmetric orbits of period two in the original dynamics,

$$
\begin{array}{ll}
00 \\
10
\end{array}, \begin{aligned}
& 10 \\
& 11
\end{aligned}
$$

one boundary orbit of original period two

and three symmetric orbits of original period four

$\begin{array}{lll}00 & 01 & 10 \\ 01 & 01 & 11 \\ 00 & 10 & 10 \\ 10 & 10 & 11\end{array}$

Note that the total number (six) is the same, but the information content is higher, since all the original period two orbits are included as period one or two, and also some orbits of original period four.

In the case $N=3$, the symmetry group of the interacting case is $C_{2}^{2}$, since there is an internal symmetry $x \rightarrow-x$, which in the coupled map survives as $(x, y) \rightarrow(-x,-y)$. This is in addition to the permutation $(x, y) \rightarrow(y, x)$. Thus the fundamental domain can be taken as $x \geq|y|$. Orbits may now be classified as asymmetric, symmetric under $(x, y) \rightarrow(y, x)$, symmetric under $(x, y) \rightarrow(-x,-y)$, symmetric under $(x, y) \rightarrow(-y,-x)$, boundary with $x=y$, boundary with $x=-y$ and (if it occurs) boundary with $x=y=0$.

\subsection{Behaviour in the coupled case}

In this section we illustrate the behaviour of the $T_{N}$ map periodic solutions when the coupling parameter $a$ is in the range $[0,0.1]$. In particular, we describe two phenomena, that is the way and stability and number of cycles change with $a$. 
Stability is expressed by the real parts ${ }^{2} \lambda_{1}, \lambda_{2}$ of the eigenvalues of the product of the Jacobian matrices

$$
J(x)=\left(\begin{array}{ll}
\frac{\partial f(x, y)}{\partial x} & \frac{\partial f(x, y)}{\partial y} \\
\frac{\partial f(y, x)}{\partial x} & \frac{\partial f(y, x)}{\partial y}
\end{array}\right)
$$

of each point of one cycle, where $f(x, y)$ and $f(y, x)$ are the set of equation (56). $\lambda$ is said to be stable when $|\lambda|<1$. When $a=0$, the systems is uncoupled and the $T_{N}$ maps have no stable cycles. Since the eigenvalues are continuous functions of $a$, we expect no stable eigenvalue to show up for very small $a$ at fixed orbit period. As for stability, we analyse the trend of the maximum and minimum eigenvalues found for each period. In general, we consider the product

$$
\Lambda=\prod_{\left|\lambda_{i}\right|>1} \lambda_{i}
$$

so that in the 2-d case it is either $\lambda_{1} \lambda_{2}$ or just the unstable $\lambda$. Stable orbits are not used for cycle expansions, hence they are not considered for this analysis. The graphs in Figure 2 show that both the maximum and the minimum eigenvalues increase hyperbolically (i.e. exponentially) with respect to the period $n_{p}$ of the cycles when $a=0$ and still up to $a \sim 10^{-7}$, but the trend of the minimum $\lambda$ 's changes as long as $a$ grows, so that their increase gets slower and slower. Besides, as we will explain later in this section, the few cycles with $|\Lambda|=4^{2 n_{p}}$ move off the fundamental domain when $a \neq 0$, so that the maximum eigenvalues take the form $|\Lambda| \leq 4^{n_{p}}{ }^{3}$ When $a=0.1$, the minimum eigenvalues are perhaps best fitted by a power law, rather than an exponential. Such a behaviour is common to all the coupled $T_{2}$ and $T_{3}$ maps. Figure 2 also shows that the trend of the minimum $\lambda$ 's also gets less and less smooth, as $a$ increases. This phenomenon, together with the loss of hyperbolicity ${ }^{4}$, dramatically slows down the convergence of cycle expansions, as we discuss in the next section. Figure 3 shows the trend of the eigenvalues in the $2 B^{-}$map when $a=0.1$. As we can see, it is very difficult to make a good fit for the trend of the minimum $|\Lambda|$ 's in this case, because all the coupled $T_{2}$ (and $T_{3}$ ) maps have got almost stable cycles for such $a$ 's, including some at high period. It is interesting to compare this behaviour with what happens with intermittent $1 D$ maps 22 .

The other interesting issue we analyse is whether and why the number of cycles of a coupled map for a fixed period varies as a function of the coupling parameter $a$. Numerical simulation shows that few periodic orbits of the uncoupled map (7) move off the fundamental domain when the system is coupled $(a>0)$. Moreover, the number of $n_{p} \geq 4$ cycles continues to decrease as $a$ grows, because some periodic solutions of the set (56) become complex or degenerate for certain values of $a$, so that most of the missing orbits have just

\footnotetext{
${ }^{2}$ henceforth we call $\lambda$ eigenvalue, but we always mean its real part.

${ }^{3}$ The same happens with the $T_{3}$ coupled maps where the maximum $|\Lambda|$ is $9^{2 n_{p}}$ when $a=0$ and $9^{n_{p}}$ or lower otherwise.

${ }^{4}$ for a hyperbolic system $|\Lambda|>A e^{b n_{p}}$ for some $A, b>0$
} 

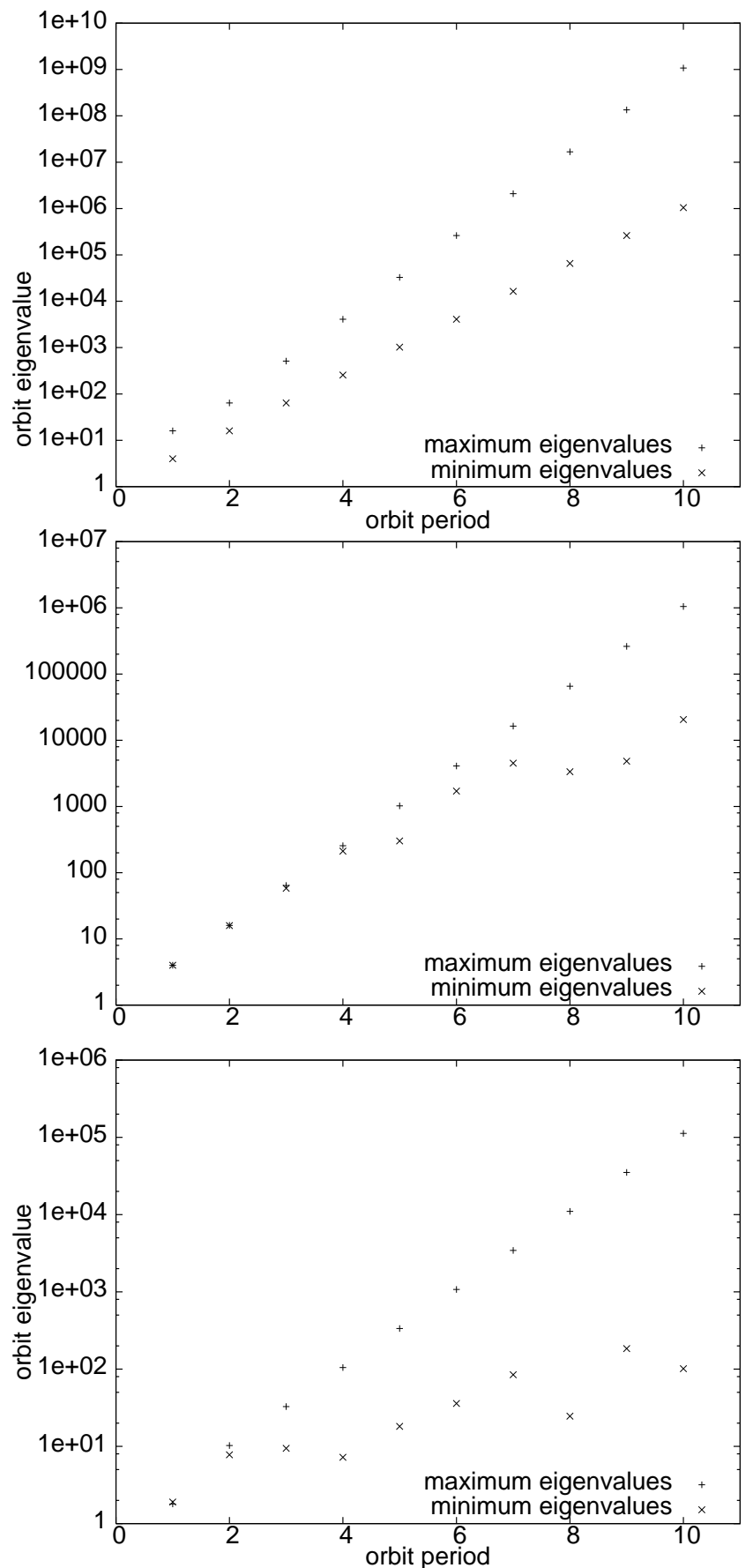

Figure 2: Trend of maximum and minimum eigenvalues of cycles up to period 10 of the $2 A^{+}$map. At the top $a=0$ (uncoupled case), in the middle $a=0.0001$, at the bottom $a=0.1$. 


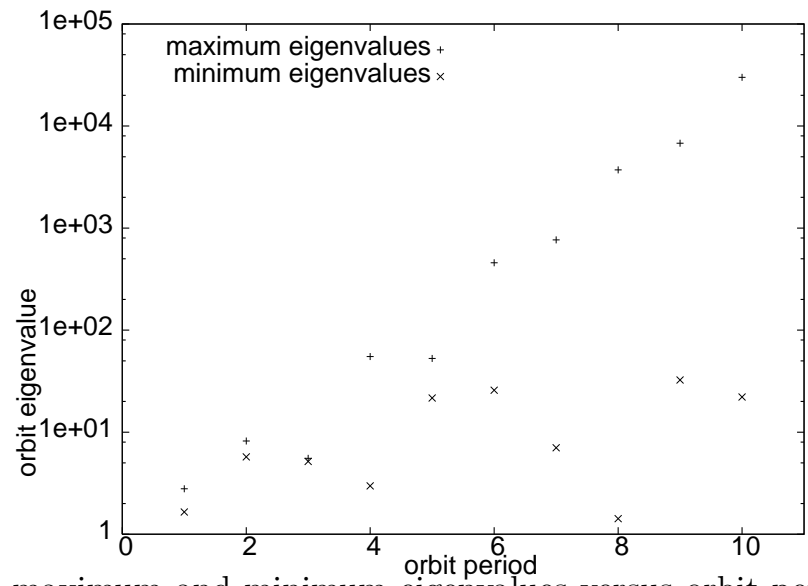

Figure 3: maximum and minimum eigenvalues versus orbit period of the $2 B^{-}$ map for $a=0.1$.

\begin{tabular}{|c|c|c|}
\hline model & $\eta$ & $C$ \\
\hline $2 A^{+}$ & 0.50 & 0.50 \\
$2 A^{-}$ & 0.50 & 0.53 \\
$2 B^{+}$ & 0.50 & 0.50 \\
$2 B^{-}$ & 0.50 & 0.54 \\
$3 A^{ \pm}$ & 0.50 & 0.61 \\
$3 B^{ \pm}$ & 0.50 & 0.63 \\
\hline
\end{tabular}

Table 1: Parameters $\eta$ and $C$ of the fitting curve (8) with $a \in\left[10^{-6}, 10^{-3}\right]$, uncertainty of about 2 on the last digit

disappeared into the complex plane. Data representing the number of periodic points $H\left(a, n_{p}\right)$ with $a \in\left[10^{-6}, 10^{-3}\right]$ have been fitted with the curve

$$
H\left(a, n_{p}\right)=N^{2 n_{p}} \exp \left(-2 n_{p} C a^{\eta}\right)
$$

where $C$ and $\eta$ are parameters that depend on the model employed, as shown in table 1 As we can see, $\eta=0.5$ in all the models, which is theoretically plausible since the dynamics and bifurcations are controlled by the critical point of the Tchebyscheff map, which is quadratic. That implies that perturbations of order $a$ have effects of order $\sqrt{a}$, hence the exponent of $0.5 . H\left(a, n_{p}\right)$ is related to the topological entropy $h(a)$ of the system by

$$
h(a)=\lim _{n_{p} \rightarrow \infty} \frac{\ln \left[H\left(a, n_{p}\right)\right]}{n_{p}}
$$

so that

$$
h(a)=2\left[\ln N-C a^{\eta}\right]
$$

which decreases with a power law as $a$ increases. Figure 4 shows good agreement of the $h(a)$ fitting function with the data for very weak coupling $\left(a \lesssim 10^{-3}\right)$ 


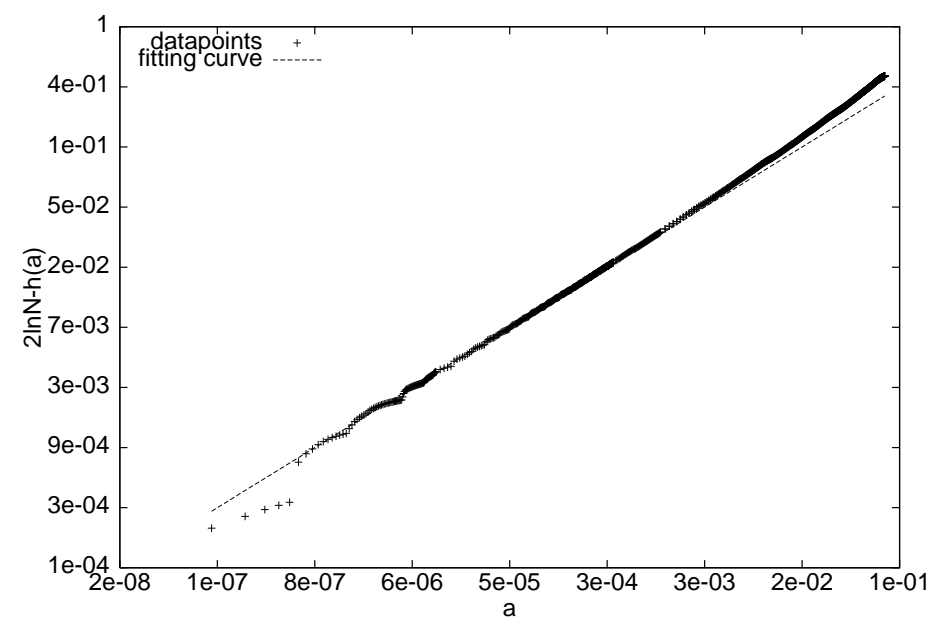

Figure 4: $\ln [2 \ln N-h(a)]$ versus $a$ in the $2 A^{+}$model.

while a slight deviation for larger $a$. This deviation is not unexpected, since this form for the topological entropy is only an approximate expression in the small coupling limit. Of course a detailed investigation could reveal that the topological entropy is not smooth in fine detail, however this form is remarkably accurate for practical purposes.

\section{3 cycle expansions and computation of averages}

\section{1 cycle expansions}

Given a quantity $v(x)$, we want to compute its average on the attractor of the dynamics (56), such as the one depicted in figure 5 . Assuming that such attractor is ergodic for any value of the coupling parameter we consider, direct simulation is the quickest method to use for this purpose, but its precision is limited by statistics. That is one reason why we use cycle expansions, which are potentially far more precise. According to periodic orbit theory [19], the average of $v$ can be found by computing the dynamical zeta function

$$
1 / \zeta=\prod_{p}\left(1-t_{p}\right), t_{p}=\frac{z^{n_{p}} e^{\beta V_{p}}}{\left|\Lambda_{p}\right|}
$$

where

$$
V_{p}=\sum_{i=0}^{n-1} v\left(f^{i}\left(x_{0}\right)\right)
$$




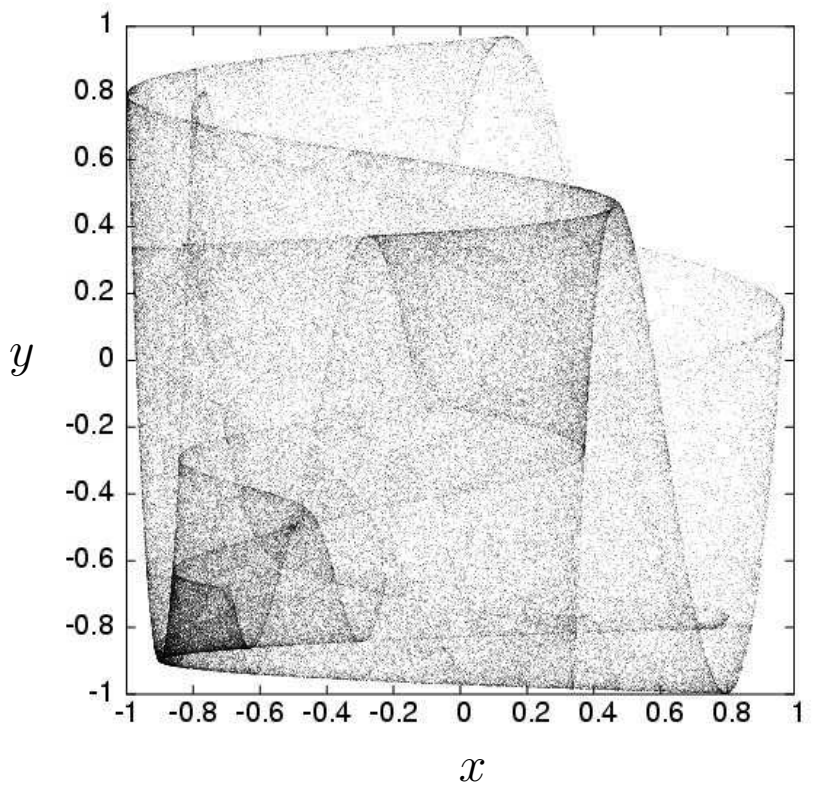

Figure 5: Scatter plot of $(x, y)$ showing the attractor of the $2 B^{+}$model at $a=0.1$.

$f$ is the map in question, $\beta$ is a parameter, $\Lambda_{p}$ the eigenvalue of the cycle $p$ and $n$ is the orbit period. The uncertain digit in the average is obtained by comparing results from the computation of (9) up to the two largest periods (for example 10 and 11). It is possible to check the rate of convergence of the zeta function by setting $\beta=0$ and $z=1$ and computing $1 / \zeta(0,0)$, which should approach zero. This way we can have an idea of how effective our cycle expansion is and whether longer cycles are needed. Figure 6 shows the convergence of $1 / \zeta(0,0)$ versus the truncation period of the expansion. As we can see, the convergence is monotonic and exponential when the coupling parameter is relatively small (say $a \lesssim 10^{-4}$ ), while it becomes nonmonotonic and increasingly slower for larger values of $a$.

The accuracy of $1 / \zeta$ is particularly affected towards $a=0.1$, where longer cycles or stability ordering may be needed. Ordering by stability means to expand the zeta function keeping all terms whose period and stability are less than a given cutoff $\left(|\Lambda|<\Lambda_{\max }\right)$. This scheme is usually preferred to length ordering in case of intermittency or bad symbolic dynamics grammar [19, but it hasn't been attempted here since it would need much a higher period of truncation for the cycle expansion and, as a consequence, impractical CPU times. A full resolution would require understanding which orbits are close to stability, which is beyond the scope of this paper.

Anyway the behaviour shown in figure [ traces back to the trend of the minimum eigenvalues of the cycles (sec. 2.3), since we can see from equation (9) that the smaller $|\Lambda|$ 's are more relevant in the computation of the zeta function. That means that if their trend is exponential with respect to increasing $a$ 's, 

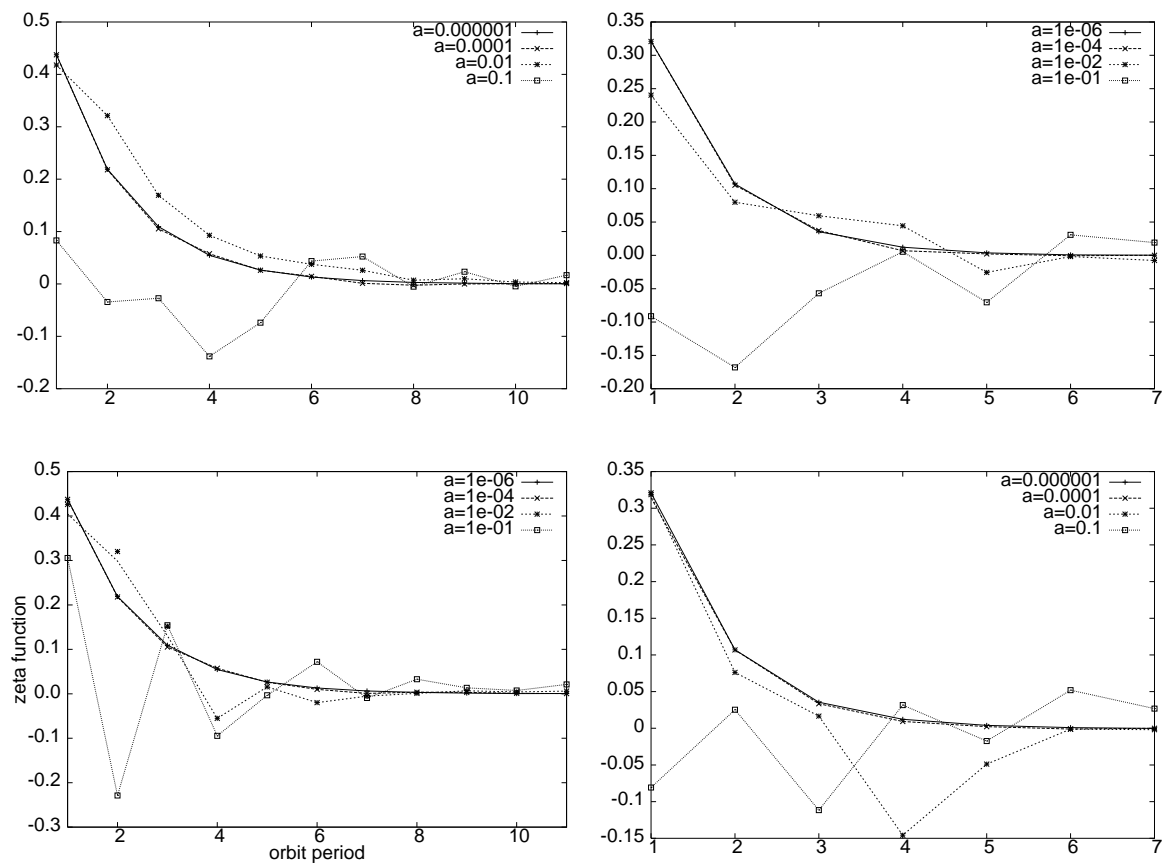

Figure 6: Convergence of $1 / \zeta(0,0)$ (logarithmic scale) versus maximum cycle length of the expansion, from the top left to the bottom right the maps $2 A^{+}, 3 A^{+}, 2 B^{+}, 3 B^{+}$, shown for different values of the coupling $a$.

the $t_{p}$ 's are smaller and smaller as $1 / \zeta(0,0)$ approaches zero. Otherwise the convergence gets more complicated. The expansion for the $T_{3}$ maps has been truncated at period 7 , yet the precision obtained is comparable with what we have got in the $T_{2}$ maps. That is because $9^{7} \approx 4^{11}$, meaning a similar number of periodic points and hence more or less the same number of terms in the two expansions.

\subsection{Computation of averages}

We now want to use cycle expansions to evaluate the average of an observable over the two-site lattice (56) and compare the outcomes with what we get with direct simulation on the same lattice. According to the theory of Beck [15], an interaction potential

$$
W(a)=\frac{1}{2} \Phi^{i} \Phi^{i+1}
$$

and self-interacting potentials

$$
\begin{gathered}
V^{(N=2)}(a)=-\frac{2}{3} \Phi^{3}+\Phi \\
V^{(N=3)}(a)=-\Phi^{4}+\frac{3}{2} \Phi^{2}
\end{gathered}
$$



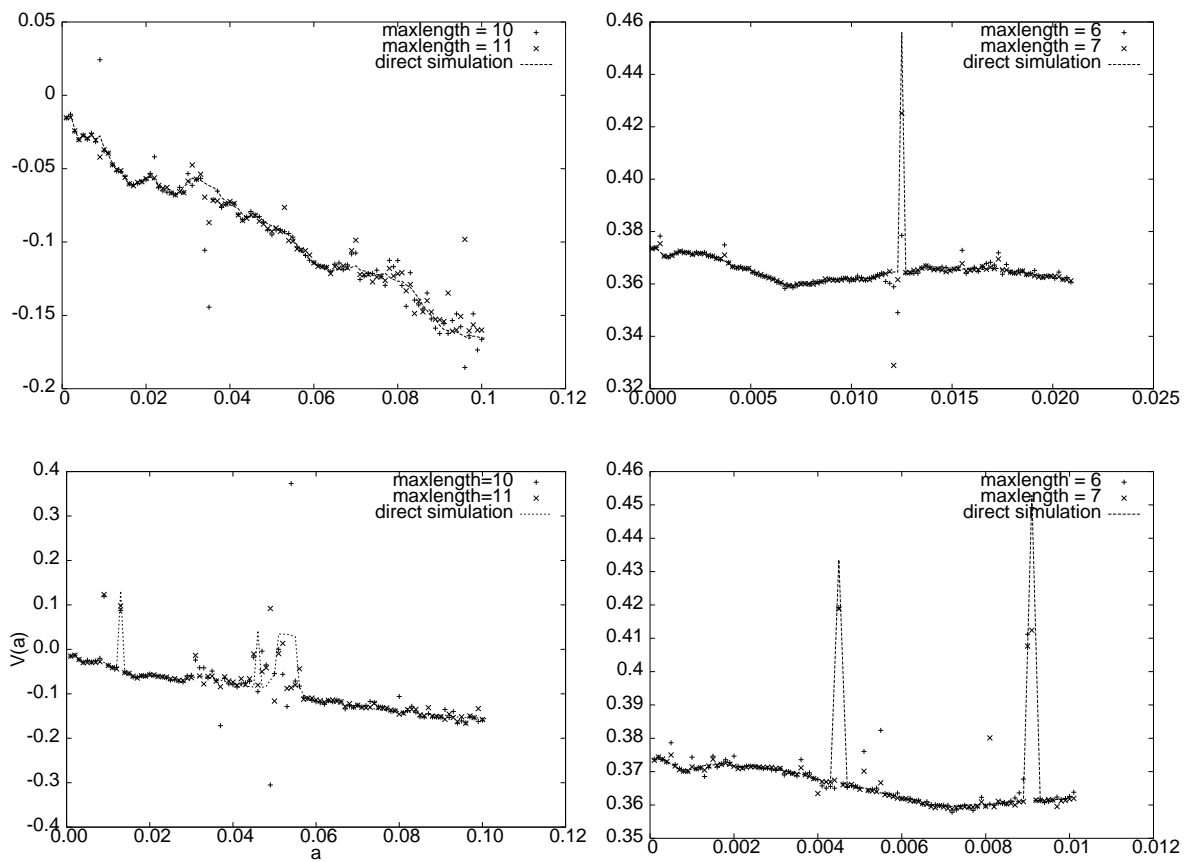

Figure 7: Average of the self-interacting potentials $V^{(2)}(a)$ for the $2 A^{+}$and $2 B^{+}$ maps (left-hand side) and $V^{(3)}(a)$ for the $3 A^{+}$and $3 B^{+}$maps (right-hand side) versus coupling parameter $a$, using the expansion (9) and direct simulation.

can be defined for the chaotic strings described by equation (4). Zeros of (11) and minima of (1213) may represent parameters of the Standard Model of particle physics such as coupling constants and masses of elementary particles. Since both the potentials defined above depend on $a$ in a nontrivial way, we can only find zeros and minima numerically by averaging both $V(a)$ and $W(a)$ over the lattice (4). Beck has obtained his results by running a long trajectory on a lattice with many sites, we are going to see what happens instead by computing the zeta function (9) on the two-site lattice (56). Figure 7 shows our results (achieved by computing (9) up to two different orbit periods) compared with direct simulation of the potentials $V^{(N)}$. A similar behaviour has been observed with the interaction potential $W(a)$. Five main features emerge.

1. In most points cycle expansion coincides with direct simulation.

2. Cycle expansion is far offset on some points, which can be explained with the presence of almost stable cycles $(|\Lambda| \sim 1)$ of high periods whose terms in eq. (9) have too much weight in the expansion.

3. There are points where both cycle expansion and direct simulation are offset. That is probably caused by one or more stable cycles or chaotic attractors, whose basins of attraction alter the phase space making it no longer ergodic. 


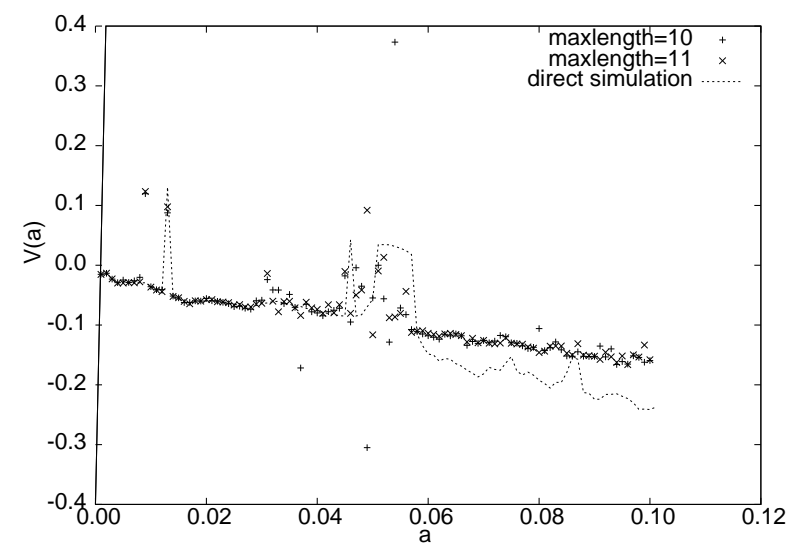

Figure 8: Average of the self-interacting potential $V^{(2)}(a)$ in the $2 B^{+}$model obtained with cycle expansion and direct simulation (with different starting values from those in figure (7) versus coupling parameter $a$.

4. The precision of the expansion visibly decreases as $a$ approaches 0.1 , as we expect from the observations made in the previous sections on the trend of the minimum eigenvalues and the behaviour of $1 / \zeta(0,0)$.

5. Figure 8 shows an offset at some point between cycle expansion and direct simulation in the model $2 B^{+}$performed with different starting values from those used in figure 7 That proves a lack of ergodicity in some regions of the interval $[0,0.1]$ of $a$ and indicates that the unstable periodic orbits are mostly unaffected by the presence of a stable orbit (or other ergodic component such as an elliptic region). Hence the cycles form a transient chaotic set more or less covering the attractor (Fig. [5) for similar values of the parameter. In this sense, the periodic orbit expansion results are more stable than direct simulation with respect to variation of the parameter, and may be preferred for this reason.

\section{Conclusions and outlook}

Periodic orbits have provided a unique window with which to view properties of weakly coupled CML. The periodic orbits in the uncoupled limit provide a reliable means of finding periodic orbits of the weakly coupled system. The symmetry of the coupled system has been exploited to improve convergence of the cycle expansion. Cycle expansion techniques work as expected, well near the uncoupled, more chaotic limit, but with slower convergence at stronger coupling. The computation of averages using cycle expansions leads to important information at a glance concerning the number and nature of nearly or fully stable states coexisting with the chaotic web of unstable periodic orbits.

The observed power law dependence of the topological entropy with parameter requires a more detailed investigation of the density and nature of bifurca- 
tions in this system to explain the value of the power $\eta=1 / 2$, the extent of its apparent universality and corrections for finite values of the parameter. For a more rigorous approach to the topological entropy of a different two site model, see 23 .

This work has clarified a number of issues and challenges regarding a more general periodic orbit approach to CML. For stronger coupling (hence weaker chaos) stability ordering of cycle expansions [19] is likely to be more effective than the length ordering employed here; this requires an understanding of the symbolic dynamics of the most stable cycles, which may be obtained in the study of the bifurcations mentioned above. Larger lattices are of course an important aspect, however the rapid decay of spatial correlations for weakly coupled CML make this less important than it might seem. The fact, as observed here, that small windows of stability at weak coupling affect the cycle expansions at only a few parameter values, gives great confidence since their presence is expected to be even less evident in lattices of larger size, in which typical orbits have many unstable directions.

\section{Acknowledgements}

We acknowledge financial support of the Nuffield Foundation (grant NAL/00353/G) and helpful discussions with P. Cvitanović. DL would also like to thank G. Servizi and V. Smith for having made available computing facilities in Bologna and Bristol respectively, as well as N. Chevalier, R. Morris and A. Shall for technical support.

\section{References}

[1] Special issue: Chaos 2, issue 3 (July 1992).

[2] K. Kaneko (Ed.) "Theory and applications of coupled map lattices" (Wiley, Chichester, 1993).

[3] Special issue: Physica D 103 (April 1997).

[4] T. Bohr, M. H. Jensen, G. Paladin and A. Vulpiani, Dynamical Systems Approach to Turbulence (Cambridge University Press, 1998).

[5] V. Baladi and H. H. Rugh, Commun. Math. Phys. 220, 561 (2001).

[6] J.-B. Bardet, Probab. Theory Relat. Fields 124, 151 (2002).

[7] A. Satake and Y. Iwasa, Ecology 83, 993 (2002).

[8] F. A. Labra, N. A. Lagos, P. A. Marquet Ecol. Lett. 6, 197 (2003).

[9] P. C. Tobin and O. N. Bjornstad, J. Anim. Ecol. 72, 460 (2003).

[10] A. O. Dudkin and V. I. Sbitnev, Biofiz. 45, 119 (2000). 
[11] R. M. C. de Almeida and M. A. P. Idiart Phys. Rev. E, 65, 061908 (2002).

[12] T. Nagatani, Physica A 289, 267 (2001).

[13] R. Tenny, L. S. Tsimring, L. Larson and H. D. I. Abarbanel, Phys. Rev. Lett. 90, 047903 (2003).

[14] S. H. Wang et al, Commun. Theor. Phys. 40, 57 (2003).

[15] C. Beck, Physica D 171, 72 (2002).

[16] C. Beck, "Spatio-temporal Chaos and Vacuum Fluctuations in Quantized Fields" (World Scientific, 2002).

[17] C. P. Dettmann, Physica D 172, 88 (2002).

[18] R. Artuso, E. Aurell and P. Cvitanović, Nonlinearity 3, (325 (1990); 3, 361 (1990).

[19] P. Cvitanović et al., "Chaos: Classical and Quantum", ChaosBook. org (Niels Bohr Institute, Copenhagen 2003).

[20] B. Lauritzen, Phys. Rev. A 43, 603 (1991).

[21] J. M. Robbins, Phys. Rev. A 40, 2128 (1989).

[22] C.P. Dettmann and P. Cvitanović, Phys. Rev. E 56, 6687 (1997).

[23] V. Afraimovich and B. Fernandez, Nonlinearity 13973 (2000). 